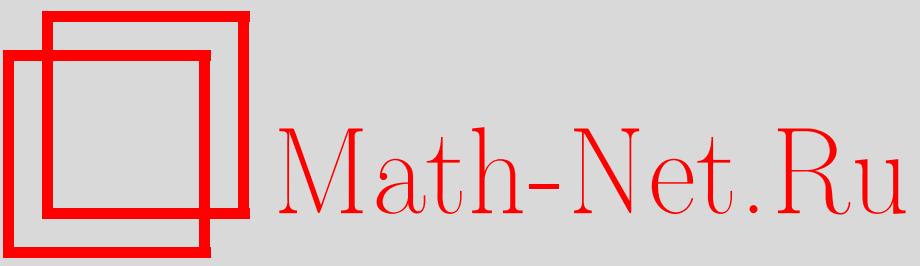

И. В. Асташова, Д. А. Соколов, О периодических решениях одной нелинейной спектральной задачи четвертого порядка, Итоги науки и техн. Сер. Соврем. мат. и ее прил. Темат. обз., 2021, том 192, 20-25

DOI: https://doi.org/10.36535/0233-6723-2021-192-20-25

Использование Общероссийского математического портала Math-Net.Ru подразумевает, что вы прочитали и согласны с пользовательским соглашением

http: //www.mathnet.ru/rus/agreement

Параметры загрузки:

IP : 54.197 .130 .99

26 апреля 2023 г., $16: 27: 45$ 


\title{
О ПЕРИОДИЧЕСКИХ РЕШЕНИЯХ ОДНОЙ НЕЛИНЕЙНОЙ СПЕКТРАЛЬНОЙ ЗАДАЧИ ЧЕТВЕРТОГО ПОРЯДКА
}

\author{
(c) 2021 г. $\quad$ И. В. АСТАШОВА, Д. А. СОКОЛОВ
}

\begin{abstract}
АннотАция. В работе доказано существование периодического решения нелинейной спектральной задачи четвертого порядка с интегральным условием.

Ключевъе слова: нелинейная задача четвертого порядка, интегральное условие, периодическое решение, задача со спектральным параметром.
\end{abstract}

\section{PERIODIC SOLUTIONS OF A FOURTH-ORDER NONLINEAR SPECTRAL PROBLEM}

\section{(c) 2021 I. V. ASTASHOVA, D. A. SOKOLOV}

\begin{abstract}
In this paper, the existence of a periodic solution to a nonlinear spectral problem of the fourth order with an integral condition is proved.

Keywords and phrases: fourth-order nonlinear problem, integral condition, periodic solution, problem with spectral parameter.
\end{abstract}

AMS Subject Classification: $34 \mathrm{C} 25$

1. Об истории вопроса. Исследуется вопрос о существовании 1-периодического решения для спектральной задачи

$$
y^{\mathrm{IV}}-|y|^{\alpha} \operatorname{sgn} y=\lambda y, \quad \alpha>1,
$$

с интегральным условием

$$
\int_{0}^{1}|y(s)|^{\alpha+1} d s=r .
$$

При оценивании первого собственного значения для различных спектральных задач для уравнений высших порядков (в том числе и нелинейных), в частности, задачи Штурма-Лиувилля с интегральным условием на потенциал, возникает вопрос о существовании решений с заданными свойствами некоторых связанных с этими задачами нелинейных задач со спектральным параметром. В [2, теорема 23], а также в [9], подобные задачи для уравнений второго порядка возникают в связи с оценкой минимального собственного значения задачи

$$
y^{\prime \prime}+\lambda Q(x) y=0, \quad x \in(0,1), \quad y(0)=y(1)=0, \quad \int_{0}^{1} Q^{\gamma}(x) d x=1, \quad \gamma \in \mathbb{R}, \quad \gamma \neq 0 .
$$

В $[1,3-5,7,12,13,17,18]$ изучены оценки первого собственного значения задачи

$$
y^{\prime \prime}+Q(x) y+\lambda y=0, \quad x \in(0,1),
$$


при различных граничных условиях и весовым интегральным условием на потенциал:

$$
\int_{0}^{1} x^{\alpha}(1-x)^{\beta} Q^{\gamma}(x) d x=1, \quad \alpha, \beta, \gamma \in \mathbb{R}, \quad \gamma \neq 0 .
$$

Классическим примером задачи четвертого порядка является задача Лагранжа об устойчивости колонны (см. [17]); другие задачи четвертого порядка исследуются в [9, гл. 6.3]. Рассматривалась нелинейная самосопряженная спектральная задача второго порядка, а также задача Эйлера-Бернулли с краевыми условиями Лидстоуна:

$$
y^{\mathrm{IV}}+q(x) y=\lambda y, \quad y(0)=y(1)=y^{\prime \prime}(0)=y^{\prime \prime}(1)=0,
$$

приводящая к постановке рассматриваемой в работе задачи. Другая задача четвертого порядка, связанная с получением экстремальных оценок собственных значений, рассматривается в [15]. Отметим, что результаты о периодических решениях нелинейных уравнений типа Эмдена-Фаулера четвертого порядка без младших членов получены в [4-8, 12, 13, 16, 18], а также в [1, § 6.4, 7.1].

\section{2. Вспомогательная теорема.}

Теорема 1. Для любого $\beta>0$ существует такое $R_{\beta}>0$, что для любого $R \in\left(0 ; R_{\beta}\right]$ найдется такая заданная на некотором отрезке $[0 ; X]$ неотрицательная функиия $v$, что

$$
\begin{gathered}
v^{\prime}(0)=v^{\prime \prime \prime}(0)=v(X)=v^{\prime \prime}(X)=0, \\
v^{\mathrm{IV}}=\left(v^{\beta}+1\right) v, \\
X^{3+8 / \beta} \cdot \int_{0}^{X} v(s)^{\beta+2} d s=R .
\end{gathered}
$$

Доказательство. Рассмотрим сначала задачу Коши с начальными условиями

$$
v(0)=h>0, \quad v^{\prime \prime}(0)=w<0, \quad v^{\prime}(0)=v^{\prime \prime \prime}(0)=0
$$

для уравнения $v^{\mathrm{IV}}(x)=f_{h}(v(x))$, где

$$
f_{h}(v)= \begin{cases}0 & \text { при } v<0, \\ v\left(1+v^{\beta}\right) & \text { при } v \in[0, h], \\ h\left(1+h^{\beta}\right) & \text { при } v>h .\end{cases}
$$

Ввиду липшицевости функции $f_{h}$ эта задача имеет единственное решение $v(x) \in C^{4}(\mathbb{R})$ при любых значениях $h$ и $w$. С помощью этого решения определим функции $v_{i}$ трех переменных, которые будут определены следующим образом: $v_{i}(h, w, x)=v^{(i)}(x), i \in\{0,1,2,3,4\}$.

Дальнейшие рассуждения основаны на знаках параметров $h>0$ и $w<0$.

Заметим, что существует такое достаточно малое $\varepsilon>0$, что $v(x)>0$ для любого $x \in[0, \varepsilon]$ и, соответственно, $v^{\mathrm{IV}}(x)=f_{h}(v(x))>0$. Отсюда следует, что $v^{\prime \prime \prime}(x)>0$ при любом $x>0$ (так как $f_{h}(v) \geqslant 0$ при всех $\left.v\right)$. Таким образом, $\partial v_{2} / \partial x>0$ при всех $h>0, w<0, x>0$.

Из неравенства $v^{\prime \prime \prime}>0$ следует существование на $(0 ;+\infty)$ не более одной точки перегиба у функции $v$. Существование же такой точки следует из того, что $v^{\prime \prime \prime}(x) \geqslant v^{\prime \prime \prime}(\varepsilon)>0$ при $x \geqslant \varepsilon$. Пусть $\xi$ - единственная положительная точка перегиба функции $v$. Ее зависимость от $h$ и $w$ задается неявно: $v_{2}(h, w, \xi(h, w))=0$. Из последнего равенства дифференцированием получаем

$$
\frac{\partial \xi}{\partial w}(h, w)=-\frac{\partial v_{2} / \partial w(h, w, \xi(h, w))}{\partial v_{2} / \partial x(h, w, \xi(h, w))} .
$$

Уже доказана положительность знаменателя в последнем выражении. Для оценки знака числителя заметим, что функция $z=\partial v_{0} / \partial w$ как функция от $x$ является решением задачи

$$
z^{\mathrm{IV}}=\frac{\partial f_{h}}{\partial v} z, \quad z(0)=z^{\prime}(0)=z^{\prime \prime \prime}(0)=0, \quad z^{\prime \prime}(0)=1 .
$$


Отсюда ввиду неравенства $\partial f_{h} / \partial v \geqslant 0$ вытекает положительность при $x>0$ функции $z$ и ее производных $z^{\prime}$ и $z^{\prime \prime}=\partial^{3} v_{0} / \partial^{2} x \partial w=\partial v_{2} / \partial w$, так что ввиду (8) получаем $\partial \xi / \partial w(h, w)<0$.

Рассмотрим два случая для значений $h$ и $w$ (не исчерпывающие все возможности).

Случай 1. $w^{2}>\frac{6 h}{5} f_{h}(h)$. Введем обозначение $x_{1}=\sqrt{-\frac{2 w}{f_{h}(h)}}>0$. Интегрируя нужное число раз неравенство $v^{\mathrm{IV}}(x) \leqslant f_{h}(h)$ (строгое при достаточно малых положительных $x$ ) и учитывая начальные условия (6), получим

$$
\begin{gathered}
v^{\prime \prime}\left(x_{1}\right)<w+\frac{x_{1}^{2}}{2} f_{h}(h)=w-w=0, \\
v\left(x_{1}\right)<h+\frac{x_{1}^{2}}{2} w+\frac{x_{1}^{4}}{4 !} f_{h}(h)=h-\frac{w^{2}}{f_{h}(h)}+\frac{f_{h}(h) w^{2}}{6 f_{h}(h)^{2}}=h-\frac{5 w^{2}}{6 f_{h}(h)}<0 .
\end{gathered}
$$

Из (9) следует, что $\xi(h, w)>x_{1}$, а так как $v$ убывает на интервале $(0 ; \xi(h, w))$, имеем $v(\xi(h, w))<$ $v\left(x_{1}\right)<0$. Таким образом, в рассматриваемом случае $v_{0}(h, w, \xi(h, w))<0$.

Случай 2. $w^{2}<\frac{h}{2} f_{h}\left(\frac{h}{2}\right)$. Введем обозначение $x_{2}=\sqrt{-\frac{2 w}{f_{h}(h / 2)}}>0$. Интегрируя нужное число раз неравенство $v^{\mathrm{IV}}(x) \geqslant 0$ (строгое при достаточно малых положительных $x$ ) и учитывая начальные условия (6), получим для всех $x \in\left(0 ; x_{2}\right]$ оценки

$$
\begin{gathered}
v(x)>h+\frac{x^{2}}{2} w \geqslant h+\frac{x_{2}^{2}}{2} w=h-\frac{w^{2}}{f_{h}(h / 2)}>\frac{h}{2}>0, \\
v^{\mathrm{IV}}(x)=f_{h}(v(x))>f_{h}(h / 2) .
\end{gathered}
$$

Интегрируя дважды последнее неравенство, убедимся, что

$$
v^{\prime \prime}\left(x_{2}\right)>w+\frac{x_{2}^{2}}{2} f_{h}(h / 2)=w-w=0 .
$$

Отсюда $\xi(h, w)<x_{2}$, так что $v(\xi(h, w))>0$. Таким образом, в рассматриваемом случае $v_{0}(h, w, \xi(h, w))>0$.

В силу непрерывности найдется такое

$$
w_{h} \in\left(-\sqrt{\frac{6 h}{5} f_{h}(h)} ;-\sqrt{\frac{h}{2} f_{h}\left(\frac{h}{2}\right)}\right),
$$

что $v_{0}\left(h, w_{h}, \xi\left(h, w_{h}\right)\right)=0$. Таким образом, при соответствующих начальных условиях, т.е.

$$
v(0)=h, \quad v^{\prime \prime}(0)=w_{h}, \quad v^{\prime}(0)=v^{\prime \prime \prime}(0)=0,
$$

точка перегиба решения $v(x)$ является также его нулем. Отметим также, что в этом случае на отрезке $\left[0 ; \xi\left(h, w_{h}\right)\right]$ функция $v$ удовлетворяет неравенству $0 \leqslant v \leqslant h$ и поэтому является решением не только уравнения $v^{\mathrm{IV}}=f_{h}(v)$, но и уравнения (4).

Покажем, что для любого $h>0$ значение $w_{h}>0$ единственно и непрерывно зависит от $h$. Для этого заметим, что производная по $w$ выражения $v_{0}(h, w, \xi(h, w))$ равна

$$
\frac{\partial v_{0}}{\partial w}(h, w, \xi(h, w))+\frac{\partial v_{0}}{\partial x}(h, w, \xi(h, w)) \cdot \frac{\partial \xi}{\partial w}(h, w) .
$$

Выше уже было показано, что $\partial v_{0} / \partial w>0$ при $x>0$ и $\partial \xi / \partial w<0$. Кроме того,

$$
\frac{\partial v_{0}}{\partial x}(h, w, \xi(h, w))<0,
$$

так как левая часть является производной в единственной точке перегиба решения $v$, имеющего отрицательные первую и вторую производные в правой полуокрестности нуля. Таким образом, выражение (13) строго положительно, что, с одной стороны, делает невозможным существование более одного $w_{h}$, удовлетворяющего равенству $v_{0}\left(h, w_{h}, \xi\left(h, w_{h}\right)\right)=0$. С другой стороны, теорема 
о неявной функции обеспечивает непрерывную (и даже класса $C^{1}$ ) зависимость $w_{h}$ от $h$. Поэтому непрерывно зависят от $h$ также

$$
X_{h}=\xi\left(h, w_{h}\right), \quad I_{\beta, h}=X_{h}^{3+8 / \beta} \int_{0}^{X_{h}} v_{0}\left(h, w_{h}, s\right)^{\beta+2} d s .
$$

Определив область возможных значений для $I_{\beta, h}$ при $0<h<\infty$, мы нашли бы те значения $R$, для которых существуют заявленные в теореме число $X$ и функция $v$.

Рассмотрим поведение $w_{h}, X_{h}$ и $I_{\beta, h}$ при $h \rightarrow 0$. Из (7) следует, что $f_{h}(h / 2)<f_{h}(h)=O(h)$, так что (11) влечет $\left|w_{h}\right|=O(h)$.

Оценим поведение $X_{h}$. Пусть $x_{*} \in\left(0 ; X_{h}\right)$ - значение $x$, при котором решение $v$ уравнения $v^{\mathrm{IV}}=f_{h}(v)$ с начальными условиями (12) принимает значение $h / 2$. Тогда

$$
\frac{h}{2}=v\left(x_{*}\right)>h+\frac{x_{*}^{2}}{2} w_{h}+\frac{x_{*}^{4}}{4 !} f_{h}(h / 2) \quad \text { или } \quad f_{h}(h / 2) x_{*}^{4}+12 w_{h} x_{*}^{2}+12 h<0,
$$

откуда

$$
x_{*}^{2}<\frac{-6 w_{h}+\sqrt{36 w_{h}^{2}-12 h f_{h}(h / 2)}}{f(h / 2)}<\frac{12\left|w_{h}\right|}{f(h / 2)}<\frac{24\left|w_{h}\right|}{h}=O(1) .
$$

Учитывая, что $v^{\prime \prime}<0$ на интервале $\left(0 ; X_{h}\right)$, можно утверждать, что в любой точке интервала $\left(0 ; x_{*}\right)$ модуль производной $\left|v^{\prime}\right|$ меньше, чем в любой точке интервала $\left(x_{*} ; X_{h}\right)$. А так как на этих интервалах приращения самого решения $v$ совпадают (оба равны $-h / 2$ ), второй интервал должен быть короче первого, т.е. $X_{h}-x_{*}<x_{*}$, откуда $X_{h}<2 x_{*}=O(1)$. Из этой оценки и неравенства $0 \leqslant v \leqslant h$ на $\left[0 ; X_{h}\right]$ вытекает, что $I_{\beta, h}=O\left(h^{\beta+2}\right)$, откуда $I_{\beta, h} \rightarrow 0$ при $h \rightarrow 0$.

Аналогично, учитывая, что $f_{h}(h)=O\left(h^{\beta+1}\right)$ при $h \rightarrow+\infty$, получим для этих больших $h$ оценки $w_{h}=O\left(h^{1+\beta / 2}\right), X_{h}=O\left(h^{-\beta / 4}\right)$ и $I_{\beta, h}=O\left(h^{-2}\right)$, откуда $I_{\beta, h} \rightarrow 0$ при $h \rightarrow+\infty$.

Таким образом, область возможных значений для $I_{\beta, h}$ при $0<h<\infty$ не совпадает с $(0 ;+\infty)$, а имеет вид $\left(0 ; R_{\beta}\right]$, где $R_{\beta}=\sup \left\{I_{\beta, h}: 0<h<\infty\right\}$. Теорема доказана.

3. Теоремы о существовании периодических решений. Перейдем к построению периодических решений.

Пусть для некоторых $\beta>0$ и $R>0$ существует, согласно теореме 1 , функция $v$, удовлетворяющая условиям (3)-(5) Зададим на отрезке $[0 ; 4 X]$ функцию $u$ с помощью соотношений

$$
u(x)=\left\{\begin{array}{cl}
v(x), & x \in[0 ; X], \\
-v(2 X-x), & x \in[X ; 2 X], \\
-v(x-2 X), & x \in[2 X ; 3 X], \\
v(4 X-x), & x \in[3 X ; 4 X] ;
\end{array} \quad u(x)=\left\{\begin{array}{cl}
v(x), & x \in[0 ; X], \\
-v(2 X-x), & x \in[X ; 2 X], \\
-v(x-2 X), & x \in[2 X ; 3 X], \\
v(4 X-x), & x \in[3 X ; 4 X] .
\end{array}\right.\right.
$$

Отметим, что в стыковых точках $X$ и $3 X$ приведенные выше разные соотношения дают одинаковые значения для первой и третьей производных функции $u$. Значения самой функции $u$ и ее второй производной в этих точках, вообще говоря, получаются с разными знаками, но, благодаря условиям (3), в обоих случаях равны нулю. В стыковой точке $2 X$, наоборот, разные соотношения приводят к одинаковым значениям функции $u$ и ее второй производной, а первая и третья производная в этой точке обращаются в ноль.

В любой точке отрезка $[0 ; 4 X]$ модули функции $u$ и ее четвертой производной совпадают с модулями функции $v$ и ее четвертой производной в соответствующей точке отрезка $[0 ; X]$. При этом знак функции совпадает со знаком ее четвертой производной. Поэтому из равенства (4) следует

$$
u^{\mathrm{IV}}=\left(|u|^{\beta}+1\right) u \quad \text { или } \quad u^{\mathrm{IV}}-|u|^{\beta} u=u .
$$

Далее заметим, что в точке $4 X$ функция $u$ и ее первая, вторая и третья производные принимают те же значения, что и в точке 0. Учитывая (14), то же можно сказать и про четвертую производную. Поэтому заданную на отрезке $[0 ; 4 X]$ функцию $u$ можно продолжить до $4 X$-периодической 
функции класса $C^{4}$ на всей прямой. При этом $4 X$ будет, очевидно, ее минимальным периодом. Кроме того, из (5) следует

$$
X^{3+8 / \beta} \cdot \int_{0}^{4 X}|u(s)|^{\beta+2} d s=4 R .
$$

Пусть теперь $n$ - некоторое целое положительное число. Введем новую функцию

$$
y(x)=(4 n X)^{4 / \beta} u(4 n X x),
$$

а также новые константы

$$
\alpha=\beta+1, \quad r=(4 n)^{4+8 / \beta} R, \quad \lambda=(4 n X)^{4} .
$$

Непосредственной подстановкой с учетом уравнения (14) получаем

$$
\begin{gathered}
y^{\mathrm{IV}}(x)-|y(x)|^{\alpha} \operatorname{sgn} y(x)= \\
=(4 n X)^{4+4 / \beta} u^{\mathrm{IV}}(4 n X x)-(4 n X)^{(\beta+1) \cdot 4 / \beta}|u(4 n X x)|^{\beta} u(4 n X x)= \\
=(4 n X)^{4+4 / \beta}\left(u^{\mathrm{IV}}(4 n X x)-|u(4 n X x)|^{\beta} u(4 n X x)\right)= \\
=(4 n X)^{4} \cdot(4 n X)^{4 / \beta} u(4 n X)=\lambda y(x),
\end{gathered}
$$

а также с учетом $(15)$

$$
\begin{aligned}
\int_{0}^{1}|y(s)|^{\alpha+1} d s=(4 n X)^{(\beta+2) \cdot 4 / \beta} \int_{0}^{1}|u(4 n X s)|^{\beta+2} d s=(4 n X)^{3+8 / \beta} \int_{0}^{4 n X}|u(t)|^{\beta+2} d t= \\
=(4 n X)^{3+8 / \beta} \cdot n \cdot 4 R X^{-3-8 / \beta}=(4 n)^{4+8 / \beta} R=r .
\end{aligned}
$$

Таким образом, функция $y(x)$ является решением задачи $(1)-(2)$. Заметим также, что функция y является 1-периодической, причем если $n=1$, то 1 является ее минимальным периодом.

При ограничении $n=1$ существование соответствующего решения доказано, учитывая формулировку теоремы 1 , только для $r \in\left(0 ; 4^{4+8 / \beta} R_{\alpha-1}\right)$. При отсутствии же ограничения можно для любого $r>0$ подобрать достаточно большое $n$, чтобы сделать $R=(4 n)^{-4-8 / \beta} r$ достаточно малым.

Итак, доказаны следующие теоремы.

Теорема 2. Для любых $\alpha>1$ и $r>0$ существует при некотором $\lambda$ периодическое решение задачи (1)-(2) с периодом 1.

Теорема 3. Для любого $\alpha>1$ существует такое $r_{\alpha}>0$, что для любого $r \in\left(0 ; r_{\alpha}\right]$ найдется $\lambda$, при котором задача (1)-(2) имеет решение $y(x)$ с наименъшим периодом, равным 1.

\section{СПИСОК ЛИТЕРАТУРЫ}

1. Асташова И. В. Качественные свойства решений квазилинейных обыкновенных дифференциальных уравнений// в кн.: Качественные свойства решений дифференциальных уравнений и смежные вопросы спектрального анализа (Асташова И. В., ред.). - М.: ЮНИТИ-ДАНА, 2012. - С. 22-290.

2. Егоров Ю. В., Кондратъев В. А. Об оценках первого собственного значения в некоторых задачах Штурма-Лиувилля// Усп. мат. наук. УМН - 1996. - 51, № 3 (309). - С. 73-144.

3. Ежак C. C., Карулина E. C., Тельнова М. Ю. Оценки первого собственного значения задачи Штурма-Лиувилля с интегральным условием на потенциал// в кн.: Качественные свойства решений дифференциальных уравнений и смежные вопросы спектрального анализа (Асташова И. В., ред.). - М.: ЮНИТИ-ДАНА, 2012. - С. 506-647.

4. Ежсак С. С., Тельнова М. Ю. Тр. семин. им. И. Г. Петровского - 2019. - 32. - С. 162-190.

5. Тельнова М. Ю. Об оценках сверху первого собственного значения задачи Штурма-Лиувилля с весовым интегральным условием// Вестн. СамГУ. - 2015. - 6 (128). - С. 124-129.

6. Astashova I. Application of dynamical systems to the study of asymptotic properties ofsolutions to nonlinear higher-order differential equations// J. Math. Sci. — 2005. — 126, № 5. — P. 1361-1391. 
7. Astashova I. V. On asymptotic behavior of solutions to a forth-order nonlinear differential equation// Proc. 1 Int. Conf. on Pure Math. (Tenerife, Spain, January 10-12, 2014). — WSEAS Press, 2014. — P. 32-41.

8. Astashova I. On asymptotic classification of solutions to fourth-order differential equations with singular power nonlinearity// Math. Model. Anal. — 2016. — 21, № 4. - P. 502--521.

9. Egorov Yu. V., Kondratiev V. A. On Spectral Theory of Elliptic Operators. - Basel, 1996.

10. Ezhak S. S. On the estimates for the minimum eigenvalue of the Sturm-Liouville problem with integral condition (English)// J. Math. Sci. — 2007. — 145, № 5. — P. 5205--5218.

11. Ezhak S. S. On estimates for the first eigenvalue of the Sturm-Liouville problem with Dirichlet boundary conditions and integral condition// in: Differential and Difference Equations with Applications (Pinelas S., Chipot M., Dosla Z., eds.). — New York: Springer, 2013. — P. 387-394.

12. Ezhak S., Telnova M. On estimates for the first eigenvalue of some Sturm-Liouville problems with Dirichlet boundary conditions and a weighted integral condition// Int. Workshop on the Qualitative Theory of Differential Equations. — Tbilisi, Georgia: Razmadze Math. Inst., 2016. — P. 81-85.

13. Ezhak S., Telnova M. On one upper estimate for the first eigenvalue of a Sturm-Liouville problem with Dirichlet boundary conditions and a weighted integral condition// Mem. Differ. Equations Math. Phys. 2018. - 73. - P. 55-64.

14. Karulina E. Some estimates for the minimal eigenvalue of the Sturm-Liouville problem with third-type boundary conditions// Math. Bohem. - 2011. - 136, № 4. - P. 377-384.

15. Karulina E. S. Oscillation properties of one fourth-order problem with a spectral parameter in boundary conditions// . Мат. Междунар. конф. «Современные проблемы математики и механики», посв. 80летию акад. В. А. Садовничего. - М.: МАКС Пресс, 2019. - С. 197-198.

16. Kiguradze I. T., Kusano T. On periodic solutions of even-order ordinary differential equations// Ann. Mat. Pura Appl. - 2001. - 180, № 3. - P. 285-301.

17. Lagrange J. L. Sur la figure des colonnes// in: Ouvres de Lagrange. — Paris: Gauthier-Villars, 1868. Vol. 2. - P. 125-170.

18. Telnova M. Some estimates for the first eigenvalue of the Sturm-Liouville problem with a weight integral condition// Math. Bohem. - 2012. — 137, № 2. - P. 229-238.

Асташова Ирина Викторовна

Московский государственный университет им. М. В. Ломоносова;

Российский экономический университет им. Г. В. Плеханова, Москва

E-mail: ast.diffiety@gmail.com

Соколов Дмитрий Андреевич

Московский государственный университет им. М. В. Ломоносова

E-mail: dmtry@nxt.ru 